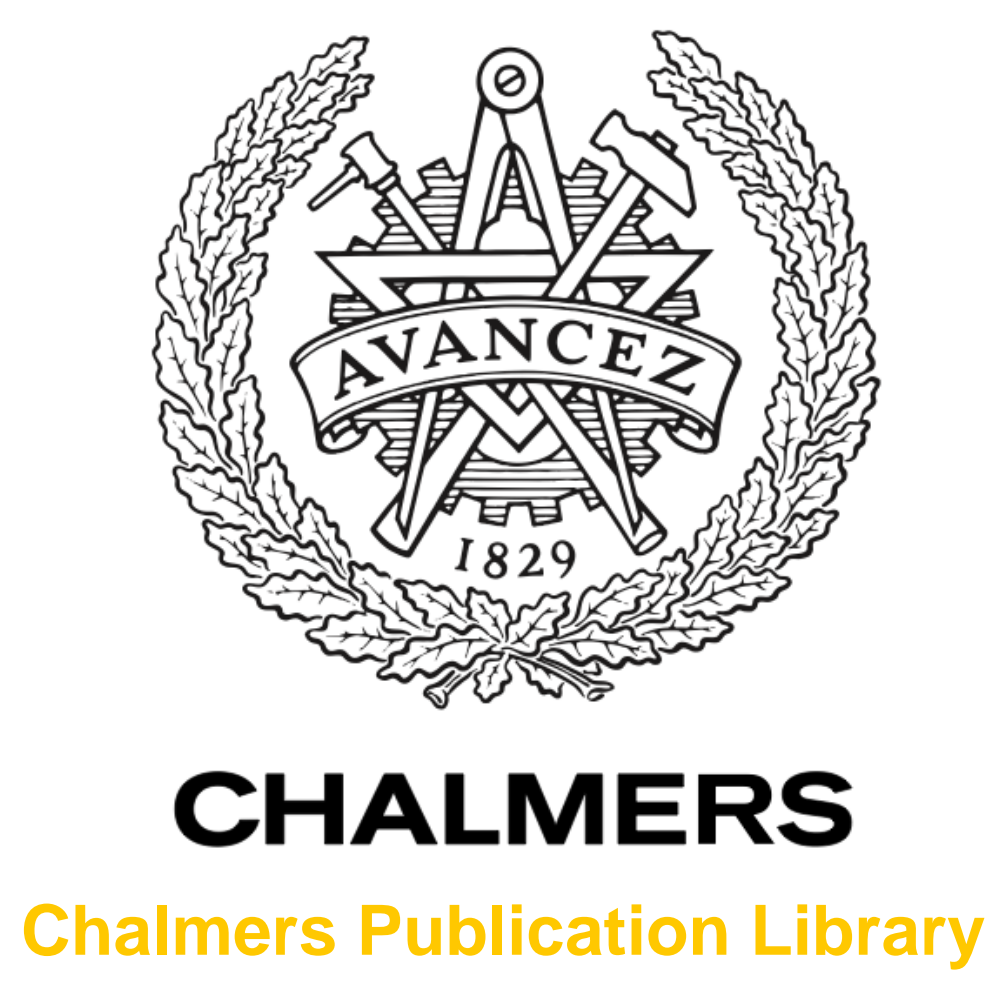

\title{
Combination of GPS and VLBI on the observation level during CONT11-common parameters, ties and inter-technique biases
}

This document has been downloaded from Chalmers Publication Library (CPL). It is the author's version of a work that was accepted for publication in:

Journal of Geodesy (ISSN: 0949-7714)

Citation for the published paper:

Hobiger, T. ; Otsubo, T. (2014) "Combination of GPS and VLBI on the observation level during CONT11 — common parameters, ties and inter-technique biases". Journal of Geodesy, vol. 88(11), pp. 1017-1028.

http://dx.doi.org/10.1007/s00190-014-0740-x

Downloaded from: http://publications.lib.chalmers.se/publication/203967

Notice: Changes introduced as a result of publishing processes such as copy-editing and formatting may not be reflected in this document. For a definitive version of this work, please refer to the published source. Please note that access to the published version might require a subscription.

Chalmers Publication Library (CPL) offers the possibility of retrieving research publications produced at Chalmers University of Technology. It covers all types of publications: articles, dissertations, licentiate theses, masters theses, conference papers, reports etc. Since 2006 it is the official tool for Chalmers official publication statistics. To ensure that Chalmers research results are disseminated as widely as possible, an Open Access Policy has been adopted.

The CPL service is administrated and maintained by Chalmers Library. 
Journal of Geodesy manuscript No.

(will be inserted by the editor)

\section{Combination of GPS and VLBI on the observation}

\section{2 level during CONT11 - common parameters, ties and}

3 inter-technique biases

\section{Thomas Hobiger . Toshimichi Otsubo}

5 Received: date / Accepted: date

6 Abstract Multi-technique space geodetic analysis software has been developed

7 which allows to combine data on the observation level. In addition to local tie information, site-wise common parameters, i.e. troposphere and clocks, can be es-

T. Hobiger

Space-Time Standards Group

National Institute of Information and Communications Technology (NICT)

4-2-1 Nukui-Kitamachi, Koganei,184-8795 Tokyo, Japan

Present address: Chalmers University of Technology, Department of Earth and Space Sciences

Onsala Space Observatory, Onsala SE-439 92, Sweden

Tel.: +46-31-772-5500

Fax: +46 31-772-5590

E-mail: thomas.hobiger@chalmers.se

T.Otsubo

Geoscience Laboratory

Hitotsubashi University

2-1 Naka, Kunitachi, Tokyo, 186-8601 Japan.

Tel.: $++81-42-580-8968$

E-mail: t.otsubo@r.hit-u.ac.jp 
9 timated with this software. Thus, it will be discussed how common parameters have to be estimated and where biases/offsets need to be taken into account. In 1 order to test such a novel concept, Global Positioning System (GPS) and Very 12 Long Baseline Interferometry (VLBI) data from the CONT11 campaign are being 13 utilized. Since the VLBI baselines of this campaign extend over several thousands 14 of kilometers, GPS data is processed in precise point positioning (PPP) mode and 15 satellite orbits and clocks are kept fixed to the IGS final products. From the obtained results it can be shown that the combination of space geodetic data on the 7 observation level leads to a consistent improvement of station position repeatability 18 as well as nuisance parameters like troposphere estimates. Furthermore, estima19 tion of common parameters (troposphere or clocks) at co-located sites helps to 20 improve the solution further and derive an utmost physically consistent model of 21 the concerned parameters.

22 Keywords GPS · VLBI · Combination · GGOS

\section{Introduction}

Space geodetic techniques are either operated at single-technique sites or they are

25 deployed at so-called co-location sites. At such stations two or more techniques

26 are operated side by side and the reference points of the individual space geodetic

27 instruments are connected by precise local tie measurements (Ray and Altamimi 2005). Thus, space geodetic data from different techniques can be combined for

29 the purpose of reducing systematic (instrumental) effects. Even if space geodetic

so techniques are analyzed with the same geophysical and mathematical models, only

31 a combination on the observation level can ensure that outliers are detected before 
32 parameters are combined, leading to a consistent usage of all observational data.

33 The establishment of such a combination process is one of the goals for the realiza-

34 tion of the Global Geodetic Observing System (GGOS) as described by Rothacher

35 et al.(2009).

36 At the moment, local tie information is used for operational combination, but

37 mostly being done either on the level of normal equations or on the level of re-

38 sults. However, recent studies from e.g. Thaller (2008), Otten et al. (2012) or

39 Coulout et al.(2007) reveal that inter-technique combination on the observation

40 level has the potential to outperform the current combination strategy. This led to

41 the formation of the International Earth Rotation and Reference Systems Service

42 (IERS) working group COL (Combination at Observation Level), which investi-

43 gates this approach in more detail.

44 In addition to the usage of local tie measurements, one can take benefit from the

45 fact that several physical and geophysical parameters, which need to be estimated,

46 are identical or only biased by a constant offset among co-located space geodetic

47 techniques. Taking advantage from these kind of "ties" allows to combine several

48 techniques more sophisticated, and to avoid that outliers or data artifacts can

49 propagate in target or nuisance parameters and, thus degrade the solution. How-

50 ever, in order to realize both, combination on the observation level and estimation

51 of common parameters a new software, which supports such approaches, had to

52 be developed. In the following sections we will follow the concept of prior studies,

53 but extend the combination not only to troposphere but also to clock parameters.

54 In addition, we will introduce new types of "ties" which relate between common

55 parameters in the case of biases between the techniques. 
${ }_{56} 2$ Space geodetic data analysis with $\mathrm{c5}++$

Driven by the need to update existing space geodetic analysis software and motivated by the demanding goals of GGOS, a new analysis package named "c $5++"$ has been developed. Other than the prior version (Otsubo and Gotoh, 2002), which was written in Java, the new software has been coded in $\mathrm{C}++$ which lead to its naming. In doing so, the software has been designed to support combination of space geodetic data from Satellite Laser Ranging (SLR), Very Long Baseline Interferometry (VLBI) and Global Navigation Satellite Systems (GNSS) on the observation level, but also enables to process single-technique solutions. As depicted in Figure 1, SLR, VLBI and GNSS modules share the same library which contains all geophysical models according to the latest IERS Conventions (Petit and Luzum 2010). In addition, local tie information can be included as virtual observations (cf. Sec. 2.1.1) which relate between the technique-specific reference points. The library also provides interfaces to various space geodetic data formats, enables reading/writing of Solution INdependent EXchange Format (SINEX) (Blewit et al., 1995) files and supports all necessary mathematical functions for the parameter adjustment process. c5++ does not have a graphical user interface (GUI) but is called directly from the command line and controlled via a configuration file. The software uses technique-specific handlers, called "players", which deals with data from a single technique. Each of these handlers, provides partial derivatives with respect to the target parameters and computes the reduced observations (OC), i.e. the difference between the observation and the computed theoretical value at that particular epoch. In addition the handler returns stochastic information (formal errors of the observations) which is being used to give weights to the data. 
As shown in Figure 1, also local ties are dealt with such a handler, which allows to apply such inter-technique information also directly on the observation level.

82 The main program calls each handler as defined in the configuration file, collects the information that is returned from each "player", sets up the design matrix and

${ }_{84}$ the stochastic model and puts all information together for parameter adjustment. In the current version of c5++, a Gauss-Markov model (Koch 1997) is used for the

86 least-squares adjustment. However, a Kalman filter (Kalman 1960) is expected to be implemented in the future as well. After the adjustment process, parameters are updated with their estimates and the main module calls again all involved handlers, which have also the capability to reject outliers. This iterative process is carried out until the ratio between the weighted root mean square error of the current iteration and the value from the prior iteration is larger than a user-defined value (in the following sections a value of 0.99 is applied). Once the iterative process is complete, the main program outputs all target parameters in SINEX format ${ }_{94}$ and provides a file that contains residuals for all data involved. c5++ has been compared against other software packages (Plank et al. 2011), and is currently being used by the Geospatial Information Authority of Japan (GSI) for ultra-rapid determination of UT1 by means of VLBI (Hobiger et al. 2010) on a routine base. In order to demonstrate the capability of the software to com- 
which share several common parameters at a co-location site, leads to a further improvement of the estimates when those additional links are applied properly.

\subsection{Combination on the observation level}

Other than combination of space geodetic results, where parameters are derived individually from each technique, combination of all available space geodetic observations on the observation level is expected to obtain more robust parameters. Outliers are less likely to bias the solution as data from other techniques helps to identify such data artifacts. Moreover, weaknesses of one technique can be compensated by adding a second technique, improving geometrical coverage and stabilizing the estimation of parameters which otherwise would depend on observations from that single technique. However, combination on the observation level does only make sense when two or more space geodetic techniques have parameters in common or their parameters can be related to each other with a mathematical model, physical relation or an external measurement, which was made at the co-location site. Local tie measurements, which fall into the latter category are in most cases the only link that relates between the different techniques. However, one can think of other ways to take benefit of co-locating space geodetic techniques. In the case of GPS and VLBI, which are both operating in the microwave band, the atmosphere around the site causes non-dispersive delays which need to be removed during the parameter estimation process. Thus, when those two techniques are co-located it is feasible to estimate a single mathematical model of the troposphere, that serves both techniques. Moreover, at many co-location sites reference signals from a frequency standard are sent to both, VLBI back-ends and 
146

GPS receivers. Thus, the same clock variation can be assumed for both techniques in principle. In the following, these three ways of tying together VLBI and GPS data on the observation level will be discussed in detail.

\subsubsection{Local ties}

For any kind of inter-technique combination, precise local tie information is necessary. Without the knowledge of local ties, space geodetic techniques could not be related to each other directly, which contradicts the idea of a co-location station. These $3 \mathrm{D}$ vectors are usually obtained from local surveys which relate the reference points of two or more space geodetic techniques to each other. After adjustment of the surveying data, the $3 \mathrm{D}$ vectors and their variance-covariance information is transformed into the terrestrial reference frame where it can be applied either on the observation level or used for combining normal equations. Local ties are provided by agencies hosting co-located instruments and are made available to the ITRF center of the IERS. Such information can be read read by c5++ directly. The software deals with this information as an independent observation, i.e. calling a dedicated handler that returns residuals (O-C) for the coordinate differences between the measured (i.e. local tie) and calculated (i.e. from the c $5++$ estimation process) inter-technique vectors. As for VLBI and GPS, this reads as

$$
\left(\begin{array}{c}
\Delta x \\
\Delta y \\
\Delta z
\end{array}\right)_{\mathrm{tie}}-\left[\left(\begin{array}{l}
x \\
y \\
z
\end{array}\right)_{\mathrm{VLBI}}-\left(\begin{array}{c}
x \\
y \\
z
\end{array}\right)_{\mathrm{GPS}}\right]=\left(\begin{array}{l}
0 \\
0 \\
0
\end{array}\right) \pm f \cdot\left(\begin{array}{c}
\sigma_{\Delta x} \\
\sigma_{\Delta y} \\
\sigma_{\Delta z}
\end{array}\right)
$$

where $\sigma_{\Delta x}, \sigma_{\Delta y}$ and $\sigma_{\Delta z}$ are the formal errors of the local tie as stated in the corresponding SINEX file. In order to give more or less weight to the local tie information it is possible to scale these formal errors by a multiplicative constant 
${ }_{172} \tau_{G P S}=m f_{h}(\varepsilon) \cdot Z H D_{G P S}+m f_{w}(\varepsilon) \cdot Z W D+m f_{g}(\varepsilon) \cdot\left(G_{N} \cos \alpha+G_{E} \sin \alpha\right)$,

$f$. As local ties are not always re-measured after changes of the station coordinates, this feature allows to maintain a certain fraction of the 3D information that relates the different space geodetic techniques to each other. However, for the analysis described in section 3 formal errors were used as they are given in the SINEX files, i.e. setting $f=1$. Such a setup is feasible although the differences between the local ties reconstructed from single-technique solutions (cf. Sec. 3.2.1) and those provided by the IERS might differ at by up to two centimeters at particular sites (e.g. CONZ and ONSA) as shown in Tab. 3). This discrepancy and and the fact that formal errors for some IERS local ties appear to be too optimistic do not play a role when combining data on the observation level, since one has to consider that local ties are introduced as virtual observations. Given the large number of GPS and VLBI observations, it turns out that even such small formal errors do not lead to a rigid inter-technique baseline vector, but still provides enough flexibility to account for technique specific systematic effects and errors in the local tie vectors. The impact of different weighting strategies, i.e. the choice of the multiplicative constant $f$ for the stochastic information of the local tie vectors, is studied separately as described in section 3.3.

\subsubsection{Common troposphere parameters}

Microwave based techniques like GPS or VLBI have in common that the neutral atmosphere (troposphere) causes signals to be delayed since the refractivity index of the gases in the media is not equal to one. Following Davis et al. (1993), one can model the troposphere excess delay in the form 
$\tau_{V L B I}=m f_{h}(\varepsilon) \cdot\left(Z H D_{G P S}+\Delta D\right)+m f_{w}(\varepsilon) \cdot Z W D+m f_{g}(\varepsilon) \cdot\left(G_{N} \cos \alpha+G_{E} \sin \alpha\right)$.

where $Z H D_{G P S}$ and $Z W D$ are hydrostatic and wet zenith delays at the GPS site and $m f_{h}(\varepsilon)$ and $m f_{w}(\varepsilon)$ denote the corresponding mapping functions which depend on the elevation angle $\varepsilon$. Horizontal gradients in North- $\left(G_{N}\right)$ and Eastdirection $\left(G_{E}\right)$ allow to consider azimuthal $(\alpha)$ asymmetry and are mapped with a dedicated gradient mapping function $m f_{g}(\varepsilon)$. Since hydrostatic delays can be computed a-priori with sufficient accuracy, one needs to estimate only wet zenith delays, respectively gradient parameters. If another microwave technique, e.g. VLBI, is co-located with the GPS antenna, one can assume that troposphere conditions are almost identical except an offset caused by different heights of the technique specific reference points. In general, any change in height is accompanied by a change of both, zenith hydrostatic delay and zenith wet delay. However, as the latter one is small compared to the hydrostatic delay effect and hydrostatic and wet mapping functions are identical at first order, it is possible to express a height shift by a change of hydrostatic delay. This can be denoted as

$$
Z H D_{V L B I}=Z H D_{G P S}+\Delta D
$$

and allows to parameterize a single zenith wet delay, i.e. $Z W D$, only. Since, horizontal gradients are assumed to be identical for co-located space geodetic instruments, VLBI troposphere delay can be modeled as$$
\text { (4) }
$$

In doing so, site dependent common troposphere parameters, i.e. $Z W D, G_{N}$ and $G_{E}$, can be estimated when data are combined on the observation level. The socalled troposphere tie $\Delta D$ can be either derived from leveling measurements and 
accurate meteorologic information (Teke et al. 2011) or estimated as an additional parameter together with the other unknowns.

\subsubsection{Common clock parameters}

Similar to common troposphere parameters which can be effectively estimated for each co-location site, the clock model can be parameterized in a similar way, if signals from a frequency standard are distributed to both systems, i.e. feeding the GPS receiver and steering the VLBI back-end. Although VLBI data are time stamped with information based on 1 pulse-per-second (PPS) signals, geodetic GPS receivers usually do not support external timing signals in the form of PPS signals. Considering only reference frequency, an unknown timing offset remains between GPS and VLBI. Moreover, un-calibrated signal paths on the way to the timing reference point of each system exist. However, as both, the oscillators of the GPS receiver and the VLBI back-end, are locked to the same reference signal, it is possible to set up a common model for clock variations and additionally estimate an inter-technique clock offset. In doing so, one can express the relation between the VLBI and the GPS clock in the form

$$
\operatorname{clock}_{V L B I}=\operatorname{clock}_{G P S}+\Delta L(t)
$$

where the last term $\Delta L(t)$ denotes the offset of the VLBI clock w.r.t. the GPS clock. If all cables and internal delays are stable or monitored one could assume $\Delta L(t)=$ const., but since this is not the case for current space geodetic timing systems, it is better to allow for a small and long-period variation of $\Delta L(t)$ (see discussion in next section). 
2.2 Prerequisites for the combination on the observation level

Although combination on the observation level is straightforward with respect to the mathematical formulation, one needs to make sure that the underlying (geo-) physical models are consistent among the different techniques. $\mathrm{c} 5++$ has been designed to ensure these requirements. However, estimation of a common troposphere model requires that a-priori hydrostatic delays are derived from consistent meteorologic models. VLBI stations are equipped with ground meteo sensors, whereas those are not always deployed at GPS sites. Such meteorologic data are usually stored only for the epochs corresponding to VLBI scans and requires temporal and spatial interpolation to meet GPS antenna locations and observation epochs. In order to consistently handle a-priori hydrostatic delays for both, VLBI and GPS, the GPT2 model (Lagler et al. 2013) is being used in this study. This ensures that troposphere ties (Eq. 3) can be estimated as daily constant offsets and do not absorb any artifacts caused by differences between measured and modeled meteorologic conditions. The concept of troposphere ties will work as long as the meteorologic conditions between two sites can be approximated sufficiently accurate by an offset $\Delta D$. This imposes an implicit constraint on the spatial distance between GPS and VLBI antennas, which should not exceed more than about 100 meter in the vertical and, depending on the topography, about one kilometer horizontally. This limit ensures that a simple troposphere bias can be estimated without the need to compensate for higher order corrections. Moreover, troposphere gradients can be assumed to be identical as long as the lateral distance between the co-located techniques does not exceed a few kilometers so that no significant changes of wet refractivity can impact the estimation process. These criteria are met for VLBI 
and GPS installations at all co-location sites, but could be problematic in the case one tries to tie DORIS (Doppler Orbitography and Radiopositioning Integrated by Satellite, (Willis et al. 2010)) sites as another microwave based space geodetic technique. Other than GPS, DORIS antennas are transmitting signals, which makes them a potential candidate for radio frequency interference (RFI) and, thus have them placed slightly away from other space geodetic infrastructures (Teke et al. 2011).

Estimation of a common clock model, that serves both, GPS and VLBI, requires that observations from both techniques are dealt with in the same time system. This requirement is fulfilled as $c 5++$ handles observations of any space geodetic technique in Coordinated Universal Time (UTC), in particular converting GPS time tags to UTC. In addition to a consistent time frame, one needs to make sure that inter-technique timing offsets are parameterized properly. Other than troposphere ties $\Delta D$, which are thought to be constant offsets over 24 hours, inter-technique clock differences $\Delta L(t)$ should be parameterized in a way that allows to consider variations at periods much longer than the temporal resolution of the clock model ( $\left.\operatorname{clock}_{G P S}\right)$. As it turns out, temperature dependent cable length variations are the dominant source for temporal changes of inter-technique clock offsets. Therefore, it is recommended to parameterize $\Delta L(t)$ so that at least a diurnal variation can be modeled properly when combining VLBI and GPS observations over a 24 hour period.

Another issue one needs to take care of, is related to the fact that VLBI sessions are not scheduled on a daily basis, sessions do not start and end at 0 UT and correlator clock models are usually not consistent among different sessions. Other than for the CONT11 data-set used here, special care needs to be taken when 
combining standard VLBI data with other observations like GPS. As c5++ can use arbitrary start and end times for the parameter estimation period all data outside periods where no VLBI data are available are therefore not considered for the adjustment process.

In summary, c5++ does not only provide the same geophysical models for different space geodetic techniques, but also utilizes only data within the same time span.

\section{Combination of GPS and VLBI during CONT11}

CONT11 was a campaign of continuous VLBI sessions, organized by the International VLBI Service for Geodesy and Astrometry (Schuh and Behrend 2007) and observed between Sep. $15^{\text {th }}$ and Sep. $30^{\text {th }}$ 2011, which were scheduled and correlated as daily sessions so that data or products can be combined with GPS over the same time span. In total 14 VLBI stations joined the CONT11 observing network. However, since not all stations continuously contributed to CONT11 and only a fraction of the network stations shared a common frequency standard with the co-located GPS receiver (Rieck et al. 2012). In order to avoid the usage of constraints for clock and troposphere parameters, the spacing of the piece-wise linear functions was selected accordingly (cf. next section). But since several participating stations had data gaps longer than those parameters intervals, such stations were excluded from the network as well, leaving only 7 stations which can be used for combination of GPS and VLBI consistently over the 15 days period. A map with the location of these stations is shown in Figure 2. 
3.1 Parameterization

As for VLBI data, c5++ provides an interface that allows to read ionosphere free observations from databases in NGS format (http://lacerta.gsfc.nasa.gov/mk5/help/dbngs_format.txt), whereas GPS observations can be input via a Receiver INdependent EXchange Format (RINEX) (Gurtner 2000) interface. In general, c5++ estimates parameters via an iterative least-squares adjustment based on a Gauss-Markov model (Koch 1997) paired with an outlier rejection based on a $3-\sigma$ criteria. Based on this approach, CONT11 data were analyzed in 24 hour batches, and different analysis options (Table 1) were selected for the computation of the target parameters. For the VLBI-only solution (S0001) no-net-translation (NNT) and no-net-rotation (NNR) constraints had to be applied in order to solve for all seven station positions without the need of fixing a single VLBI site to its nominal ITRF2008 coordinates (Altamimi et al., 2011). Stations TSKB and CONZ were excluded from the NNR/NNT conditions in order to account for site displacements caused by large Earthquakes. The GPS-only solution (S0010) was obtained from un-differenced observations which were processed in static precise-point positioning (PPP) mode (Kouba and Heroux, 2001), while using IGS final orbit and clock products (Dow et al. 2009). The PPP mode allows us to process the observations independently from the length of the baselines defined by the VLBI network geometry. However, the small number of stations involved in this study does not allow an estimation of satellite orbit and clock parameters. An elevation cut-off angle of 5 degrees was applied to all GPS sites and ambiguities were solved as floats. Compared to the VLBI data-set, GPS observations provide a better geometrical and temporal coverage, which allows to solve for station clock parameters with a finer resolu- 
tion, i.e. estimating piece-wise linear clock models with nodes every 5 minutes (cf.

Table 1). The first solution which combines GPS and VLBI on the observation level, i.e. S0011, only added local tie vectors as virtual observations that relate between the reference points of the co-located VLBI and GPS antennas. Taking this concept a step further leads to solution S0111, which includes the estimation of site-dependent troposphere parameters, i.e. zenith wet delays and gradients, rather than estimating such parameters for VLBI and GPS separately. The last solution (S1111) extends the parameterization of S0111 and deals with clock estimates as site-dependent parameters as well. This can be realized by considering a slowly varying inter-technique electrical cable delay change which is being modeled by a continuous piece-wise linear function with a temporal resolution of 3 hours. In all solutions earth orientation parameters (EOPs), i.e. UT1 and pole coordinates $\left(X_{p} / Y_{p}\right)$, were only estimated from VLBI data since GPS orbits were kept fixed to their IGS values. Tab. 2 lists the geophysical models used for the computation of single-technique and combined solutions.

Weighting of data and virtual observations, e.g. local ties, is not a straightforward problem and is usually handled by variance component estimation (Kelm, 2009). However, as this feature is currently not implemented in the $c 5++$ framework one has to rely on the weights deduced from the formal errors from each measurement type. As for VLBI, formal errors provided from the correlator are taken and multiplied with the wet mapping functions in order put lower weight on low elevation observations. GPS code and carrier phase observations are assumed to have formal errors of $70 \mathrm{~cm}$ and $7 \mathrm{~mm}$, respectively. Also these formal errors are multiplied with the wet mapping function coefficients in order to account for a decrease of precision at lower elevations. As mentioned before, local tie vectors 
are introduced with the formal errors provided in the IERS SINEX files. These uncertainties might be too optimistic (cf. Tab. 3) but one has to consider that local tie vectors are introduced on the observation level like any other observable. As three virtual observations, i.e. one for each local tie coordinate component, compete against thousands of GPS and VLBI observations, it is feasible to assume that even though the formal errors are too optimistic the combined solution is not constrained to a rigid local tie vector which is purely determined by the IERS local tie information. The impact of the weight with which these local ties are introduced has been studied separately and is summarized in section 3.3.

\subsection{Results}

In order to judge whether combination on the observation level improves the estimation of geodetic target parameters, station coordinates are studied in the following. In addition, nuisance parameters, in particular troposphere estimates, which are also used outside the geodetic community, are compared. Moreover, the feasibility of estimating a station-wise common clock model, respectively parameterizing clock-ties with a long-term variation, is being reviewed.

\subsubsection{Site coordinates}

Station position repeatabilities, measured as root mean squared (RMS) error of the offset-removed coordinate time series, during the 15 day period are computed for each solution and plotted for individual stations in Figure 3. In addition, average RMS values over all network sites are summarized in Table 4. VLBI-only station position repeatability is worse than the GPS-only solution. In general, scattering of 
the VLBI-only solution is about twice as large as the GPS single-technique position results. However, once data are combined on the observation level and local tie vectors are added as virtual observations, co-located VLBI and GPS sites reveal the same stability. Thus, in the following only repeatabilities of the GPS receivers will be discussed, although identical conclusions could be drawn also from the VLBI coordinate time series of any combined solution. One can see that adding VLBI and local tie-information improves the station position repeatabilities of the GPS sites. Only at Hartebeesthoek, South Africa (GPS:HRAO/VLBI:HARTRAO) a small degradation of the performance can be noticed. Estimating the troposphere conditions as site-dependent common parameters, as done in solution S0111, has another positive impact on the station position repeatability. Although the total number of VLBI scans is relatively small compared to all GPS observations, most of the VLBI data is taken at very low elevation angles, given the long baselines and the mutual source visibility. This allows to better de-correlate station position, clock and troposphere parameters and helps to overcome drawbacks which GPS is facing due to antenna phase center variations. Finally, the estimation of both, common troposphere and clock parameters, as carried out in solution S1111, yields the best performance among all solution strategies. However, as discussed in section 2.2 one can not simply assume a constant offset between the VLBI and GPS timing equipment, but needs to model at least a time dependent inter-technique clock offset. Although improvements are at the sub-mm level, it is clearly indicated that combination on the observation level, respectively estimating common parameters (troposphere, clocks), has a positive influence on the stability of the obtained coordinate time series. 
Beside traditional space geodetic products like station position, troposphere estimates are being used for various purposes outside the geodetic community. Thus, the impact of combination on the observation level, respectively the estimation of site-dependent common troposphere models, is being investigated. As an example, troposphere estimates at station Wettzell, Germany during CONT11 are shown in Figure 4. Single-technique troposphere estimates and solution S0011, which combines GPS and VLBI but does contain a station-wise common troposphere model, reveal a few spikes in the time series, which are likely caused by undetected outliers which got absorbed into the troposphere estimates. In particular the VLBI estimates seem to suffer from this effect. However, as soon as station-dependent common troposphere models are estimated, i.e. solutions S0111 and S1111, those data artifacts do not mitigate into the troposphere parameters. In addition, daily estimates of troposphere ties $\Delta D$ (see discussion in Section 2.1.2) are stable over time with $\pm 2 \mathrm{~mm}$. Table 5 lists the estimated station-dependent troposphere ties and compares them with theoretical values from Teke et al. (2011). Except for stations KOKB and WES2, estimated troposphere ties agree well with the expected values derived from height differences and average atmosphere conditions. The estimates for WES2 are consistent with the value derived by Thaller (2008), but differences at KOKB remain unexplained. The most likely explanation for differences between estimated and empirically modeled troposphere ties might be given

403 by antenna radome or multi-path effects. 
Estimating a single clock model for both techniques at each site, significantly reduces the number of unknowns and, thus helps to stabilize the solution, respectively makes it easier to detect outliers, which would otherwise propagate to a large extent into the clock solution. Although, a common clock model improves the estimation of the target parameters, as discussed in the prior sections, the assumption that clock ties $\Delta L(t)$ can be modeled by a piece-wise linear function, with a time resolution of 3 hours, is crucial. If reference frequency signals are distributed perfectly to VLBI and GPS components, one would expect that $\Delta L(t) \approx$ const. within the formal error of the estimates. However, when computing the RMS of the de-trended clock-tie estimates (Fig. 5) it obvious that several stations have inter-system delay variations which exceed the average formal error of these biases. In particular stations at which GPS and VLBI technology are separated further away, e.g. the Transportable Integrated Geodetic Observatory (TIGO) in Concepcion and Tsukuba (TSKB), it is not feasible to estimate a common clock without considering intra-day variations of the clock-tie. On the other side, at stations where the GPS antenna is located close to the VLBI facilities (e.g. at Onsala (ONSA)), almost no significant sub-daily inter-technique delay variations are found. This sustains the hope that in the future, more stable and well monitored frequency distribution systems become commercially available, so that VLBI and GNSS technology can be locked to a single frequency standard and inter-system delays, i.e. clock ties, are reduced to a single constant offset, which can be estimated with the other unknown parameters. 
3.3 On the impact of different weights for the local ties

Introducing local tie information with the stochastic information provided in the

SINEX files could lead to too tightly coupling of co-located site. For example, formal errors of $0.1 \mathrm{~mm}$ for the local tie at WTZZ might be too optimistic for the description of the physical accuracy of that inter-technique baseline vector. As mentioned in the prior sections, station position repeatabilities of VLBI were almost identical to those of GPS. This confirms the concept that co-located stations are allowed only identical movements, but bears the risk of constraining on technique, i.e. VLBI, closer to the technique which dominates the solution (i.e. GPS) because of its larger number of observations.

In order to test the impact of the stochastic information for the local tie vectors, solutions S0011 and S1111 were computed with different choices for the multiplicative constant $f$ (cf. Eq. 1). Other than in the analysis before, where local ties were introduced with the formal errors given in the SINEX files, i.e. $f=1$, also lower weights for the uncertainty of these links were tested with $f=2,4,8,16$. Mean $3 \mathrm{D}$ station position repeatabilities were then taken as criteria in order to judge how the choice of the local tie uncertainty impacts each solution. One expects that lower weights for the local ties lead to less coupling of the obtained station positions and thus yield more independency among the station positions of the different space geodetic techniques.

As shown in Figure 6 this assumption is confirmed. In general, lower weights, i.e. larger values of $f$, lead to more scattering of the station coordinates of a single technique, in particular VLBI. This is clearly visible from solutions S0011, which rely only on local tie information that relates between both techniques. A similar 
451 pattern can be seen for solutions S1111, but here one notices that implicit ties

from common troposphere and clock models help to reduce such a degradation. In general, it can be concluded that lower weights for the local ties lead to a performance which is closer to those of single-technique solutions when no other common models, either troposphere or clock, are estimated together. Moreover, one needs to consider that too loose constraints bear the risk that VLBI station position scattering gets worse than the single technique solution since the VLBI network has not been constrained by NNR/NNT conditions, but purely relies on the local tie information which implicitly orients and aligns the VLBI network stations.

Similar to the results discussed in section 3.2 .1 one can observe only sub-mm changes of the GPS station coordinate repeatabilities, but sees a large impact on the VLBI station position performance, which is clearly benefiting from closer ties with the co-located GPS receiver. Although the impact of the combination of the observation level leads only to small improvements for the GPS station coordinate repeatabilities, the benefit of this approach can be confirmed consistently every time VLBI and GPS are analyzed together.

For the future a significant improvement is expected after next generation VLBI technology (Petrachenko et al. 2012) is in place. Such new technology is expected to produce more scans and thus enable VLBI to compete better with the large number of GPS measurements. In addition, a variance component estimation, which includes the formal errors of the local ties, could help to include these intertechnique vectors in the adjustment process with more realistic weights. 
It could be shown that combination of space geodetic data on the observation level improves both, geodetic target parameters and nuisance parameters like troposphere estimates. Parameters estimated from the combined approaches performed better than any of the single-technique solutions. In addition, outliers are less likely to mitigate into parameters when more than one space geodetic technique is used to estimate physically identical quantities like troposphere or clock offset. However, special care needs to be taken when tying two or more techniques together, by means of other than geometrical information, i.e. locally measured $3 \mathrm{D}$ vectors. If the troposphere is used as a proxy for tying together microwave based space geodetic techniques, both the underlying physical model as well as the functional representation in the adjustment process, are consistent if a so-called tropospheretie, which corrects for different station heights (respectively zenith delays), is taken into account. Such a troposphere tie can be applied a-priori, if accurate meteorologic information is available at all co-location instruments. Otherwise, one can parameterize troposphere-ties as inter-system troposphere biases in the adjustment process. The latter approach was pursued here, leading to estimates which match with empirically derived troposphere ties within the formal uncertainties. However, GNSS antenna radome and multi-path effects can be absorbed into troposphere ties, leading to estimates which can not by assigned to differential zenith troposphere delays only.

Estimation of a common clock model, as a third way of tying together different space geodetic techniques, is feasible as well. However, special care needs to be taken, since intra- and inter-system delay changes do not allow to estimate a sim- 
ple constant bias for the duration of a 24 hour session. Since most cable length changes and internal delay variations are strongly correlated to temperature variation, clock-ties have to be parameterized in a way which accounts at least for diurnal variations.

The network of 7 stations likely does not reveal the full potential of this approach and further studies concerning the suggested combination approach are needed to confirm the benefits presented here. However, we find a consistent improvement each time we add another parameter to be estimated from both techniques, which would not be the case if combination of common parameters on the observation level does not work or has conceptual errors.

\section{Outlook}

Space geodetic techniques are currently not only improved concerning measurement precision, but also undergo system upgrades which allow to obtain more observations per session. In the case of GNSS, this is achieved by the inclusion of more satellite systems. However, even within GNSS, biases between the different systems exist. Thus, before combining GNSS with other space geodetic techniques, such biases need to be well understood and either compensated or estimated properly. As for VLBI, it is envisaged that the VLBI2010 system will replace the current S/X-band systems in the next several years (Petrachenko et al. 2012). With the introduction of phase delay observables, a significant improvement in measurement precision is expected from this new technology, making it more competitive against GNSS and SLR.

Combination of space geodetic techniques implies that local ties are well known, 
monitored and are made accessible for the analysts. Thus, local tie surveys need to be carried out more regularly and accuracy of the inter-technique links should be better than the performance of space geodetic techniques, respectively the GGOS goals. If one wants to take benefit from common frequency standards, it is recommended to monitor cable delay changes and calibrate internal delay variations. Estimation of such biases seems to be feasible as shown in this study, but knowledge of relative or even absolute timing offsets will likely improve the concept of combining space geodetic techniques by considering a common clock model. First efforts to establish such a system for VLBI are currently ongoing as reported by Panek et al. (2013).

In general, one can extend the concept of combination on the observation level and include a third space geodetic technique, e.g. SLR, and estimate orbit parameters as well. In doing so, GPS satellites which are also tracked by SLR sites would increase the number of implicit links between the techniques and further improve the estimation of target parameters. Although c5++ would support such an approach after minor updates, one needs to improve the mathematical concept of the estimation process as the huge number of unknowns, which have to be solved in a single adjustment process, likely poses a problem with the current estimation method. In addition, one needs to reconsider how observational data from different space geodetic technique should be weighted. As VLBI and GPS do not contribute the same number of observations during a $24 \mathrm{~h}$ session, one needs to improve the mathematical concept behind the estimation method. In principle this can be achieved by a variance component estimation as suggested by Kelm (2009). This approach has not been used for this study, but might be applicable in future investigations. 
In general, the combination of space geodetic data on the observation level appears to be a promising strategy to support the GGOS goals and help to realize the next generation of reference frames which are required for monitoring global change.

Acknowledgements Parts of this work were supported by a Grant-in-Aid for Scientific Research. The International GNSS Service (Dow et al. 2009) and the International VLBI Service for Geodesy and Astrometry (Schuh and Behrend 2011) are acknowledged for providing data.

Design and implementation of c5++ was a huge effort, requiring the participation and support of many individuals.

\section{References}

1. Altamimi Z, Collilieux X, and Metivier L (2011) ITRF2008: an improved solution of the international terrestrial reference frame, J Geod 85(8):457-473, doi:10.1007/s00190-011-04444

2. Chen G, Herring TA (1997) Effects of atmospheric azimuthal asymmetry on the analysis of space geodetic data. J Geophys Res 102(B9):20489-20502. doi:10.1029/97JB01739

3. Coulot D, Berio P, Biancale R, Loyer S, Soudarin S, Gontier AM (2007) Toward a direct combination of space-geodetic techniques at the measurement level: Methodology and main issues, J Geophys Res, 112(B5):2156-2202 doi:10.1029/2006JB004336

4. Davis JL, Elgered G, Niell AE, Kuehn CE (1993) Ground-based measurements of gradients in the "wet" radio refractivity of air, Radio Science, 28(6), 1003-1018, doi:10.1007/s00190011-0444-4

5. Dow JM, Neilan RE, Rizos C (2009) The International GNSS Service in a changing landscape of Global Navigation Satellite Systems, J Geod 83(3-4),191-198, doi:10.1007/s00190008-0300-3

6. Gambis D (2004) Monitoring Earth orientation using space-geodetic techniques: state-ofthe-art and prospective., J Geod 78(4,5):295-303, doi:10.1007/s00190-004-0394-1.

7. Gurtner W (2000) RINEX: The Receiver-Independent Exchange Format, GPS World, $5(7): 48-52$ 
8. Hobiger T, Otsubo T, Sekido M, Gotoh T, Kubooka T, Takiguchi H (2010) Fully automated VLBI analysis with c5++ for ultra-rapid determination of UT1, Earth Planets Space 45(2):75-79, doi:10.5047/eps.2010.11.008.

9. Hobiger T, Otsubo T, Sekido M (2014) Combination of space geodetic observations from SLR and VLBI on the observation level with c5++, Adv Space Res 53(1):119-129, doi:10.1016/j.asr.2013.10.004.

10. Kalman RE (1960) A New Approach to Linear Filtering and Prediction Problems, Transactions of the ASME-Journal of Basic Engineering, 82(D):35-45.

11. Kelm R (2009) Rigorous Variance Component Estimation in Weekly Intra-Technique and Inter-Technique Combination for Global Terrestrial Reference Frames, International Association of Geodesy Symposia, Springer Berlin Heidelberg, 134, 39-44, doi:10.1007/978-3-64200860-3_6.

12. Koch KR (1997) Parameter Estimation and Hypothesis Testing in Linear Models, Springer, Berlin.

13. Kouba J, Heroux P (2001), Precise Point Positioning using IGS orbit and clock products, GPS Solutions 5(2):12-28, doi:10.1007/PL00012883

14. Lagler K, Schindelegger M, Boehm J, Krasna H, Nilsson T (2013) GPT2: Empirical slant delay model for radio space geodetic techniques, Geoph Res Lett 40: 1069-1073, doi: $10.1002 /$ grl.50288

15. Nilsson T, Boehm J, Schuh H (2011) Universal time from VLBI single-baseline observations during CONT08, J Geod 85(7): 415-423, doi:10.1007/s00190-010-0436-9.

16. Otsubo T, and Gotoh T (2002), SLR-based TRF Contributing to the ITRF2000 project, IVS 2002 General Meeting Proceedings, 300-303, 2002.

17. Otten M, Flohrer C, Springer T, Enderle W (2012) Multi-technique combination at observation level with NAPEOS: combining GPS, GLONASS and LEO satellites, Geophysical Research Abstracts, 14, EGU2012-7925-1.

18. Panek P, Kodet J, Prochazka I (2013) Accuracy of two-way time transfer via a single coaxial cable, Metrologia, 50(1):60-65, doi:10.1088/0026-1394/50/1/60.

19. Petit G, Luzum B (2010) IERS Conventions (2010) IERS Technical Note, 36, 179 pp. 
20. Petrachenko WT, Niell AE, Corey BE, Behrend D, Schuh H, Wresnik J (2012) VLBI2010: Next Generation VLBI System for Geodesy and Astrometry, Geodesy for Planet Earth - International Association of Geodesy Symposia, 999-1005, doi:10.1007/978-3-642-20338-1_125.

21. Plank L, Boehm J, Schuh H (2011), DeDeCC - Comparison of VLBI data analysis software - results, 12th IVS Analysis Workshop, March 31, 2011, Bonn, Germany, http://amalthea.hg.tuwien.ac.at/ members/DeDeCC/Comparison_Campaign.pdf.

22. Ray J, Altamimi Z (2005) Evaluation of co-location ties relating the VLBI and GPS reference frames, J Geod 79(4-5): 189-195, doi:10.1007/s00190-005-0456-z

23. Ray RD, Ponte RM (2003) Barometric tides from ECMWF operational analyses. Ann Geophys 21(8):1897-1910. doi:10.5194/angeo-21-1897-2003

24. Rieck C, Haas R, Jarlemark P, Jaldehag K (2012) VLBI frequency transfer using CONT11, European Frequency and Time Forum (EFTF), 163-165, doi:10.1109/EFTF.2012.6502358.

25. Rothacher M, Beutler G, Bosch W, Donnellan A, Gross R, Hinderer J, Ma C, Pearlman M, Plag HP, Richter B, Ries J, Schuh H, Seitz F, Shum CK, Smith D, Thomas M, Velacognia E, Wahr J, Willis P, and Woodworth P (2009), The future Global Geodetic Observing System (GGOS), in The Global Geodetic Observing System: Meeting the Requirements of a Global Society on a Changing Planet in 2020, Plag and Pearlman (eds.), Geoscience Books, Springer Berlin.

26. Schuh H, Behrend H (2012), VLBI: A fascinating technique for geodesy and astrometry, J Geody 61:68-80, doi:10.1016/j.jog.2012.07.007

27. Teke K, Boehm J, Nilsson T, Schuh H, Steigenberger P, Dach R, Heinkelmann R, Willis P, Haas R, Garcia-Espada S, Hobiger R, Ichikawa R, Shimizu S (2011) Multi-technique comparison of troposphere zenith delays and gradients during CONT08, J Geod 85(7):395413, doi:10.1007/s00190-010-0434-y.

28. Thaller D (2008) Inter-technique combination based on homogeneous normal equation systems including station coordinates, Earth orientation and troposphere parameters; Scientific Technical Report STR 08/15, Deutsches GeoForschungsZentrum, ISSN 1610-0956.

29. Willis P, Fagard H, Ferrage P, Lemoine FG, Noll CE, Noomen R, Otten M, Ries JC, Rothacher M, Soudarin L, Tavernier G, Valette JJ (2010) The International DORIS Service, Toward maturity, Adv Space Res, 45(12):1408-1420, doi:10.1016/j.asr.2009.11.018. 


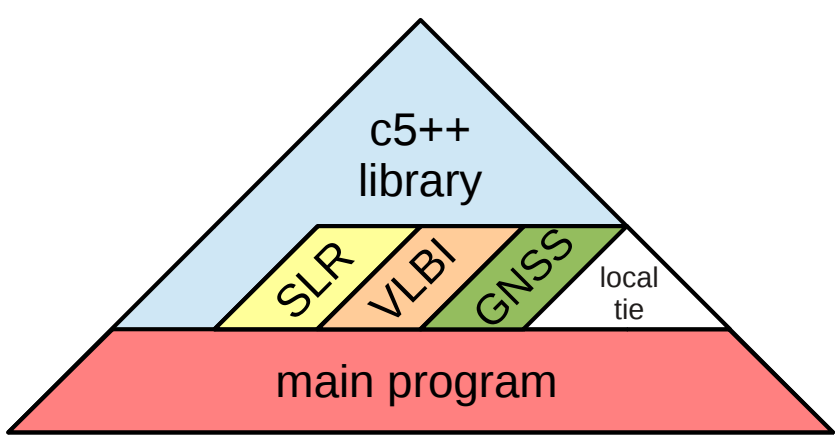

Fig. 1 The basic concept of c5++ allows to process single- and multi-technique space geodetic observations by taking advantage from the usage of identical geophysical models. 


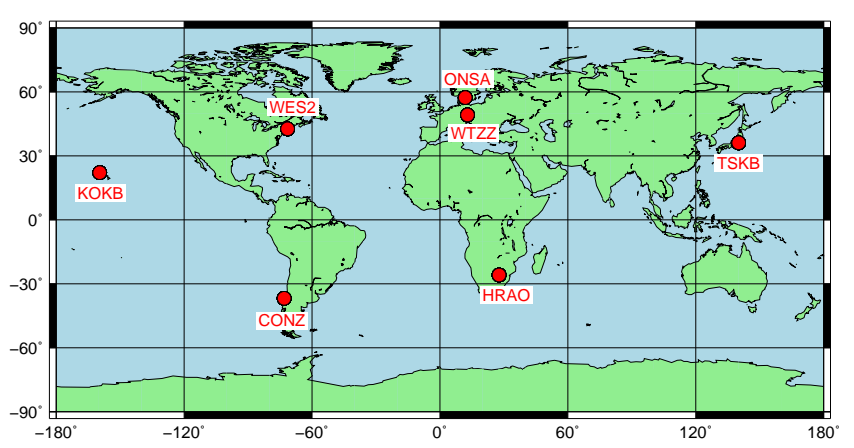

Fig. 2 Location of all stations which participated in the CONT11 campaign and were considered for combination on the observation level. Sites are abbreviated with their IGS name, i.e. Hartebeesthoek (HRAO), Kokee Park (KOKB), Onsala (ONSA), TIGO Concepcion (TIGO), Tsukuba (TSKB), Westford (WES2) and Wettzell (WTZZ). 


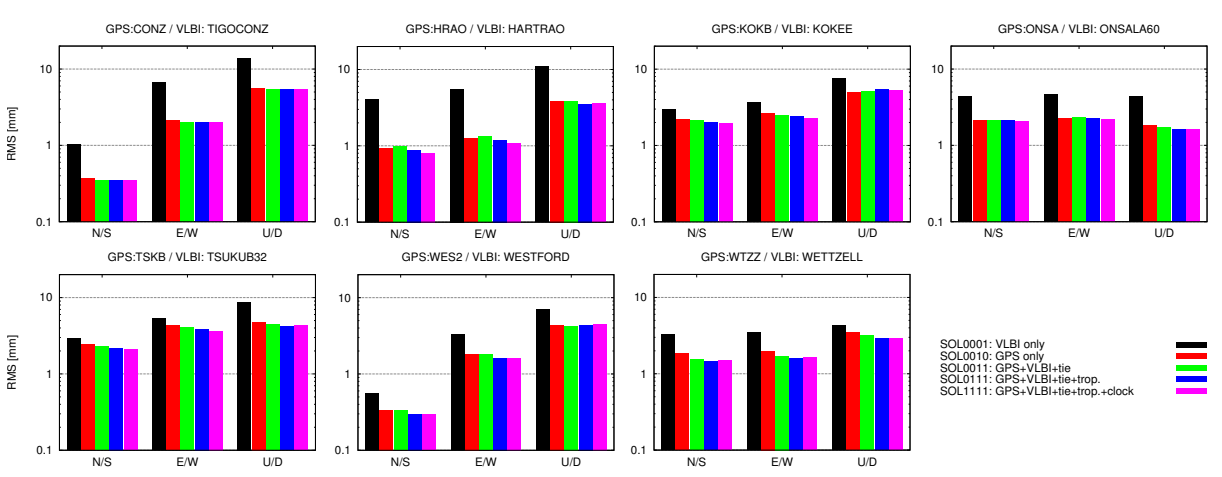

Fig. 315 days station position repeatabilities, measured as root mean square (RMS) error,

from each solution are plotted for individual stations. Ordinates are scaled logarithmically for better readability. Average RMS values over all network sites are summarized in Table 4 . 

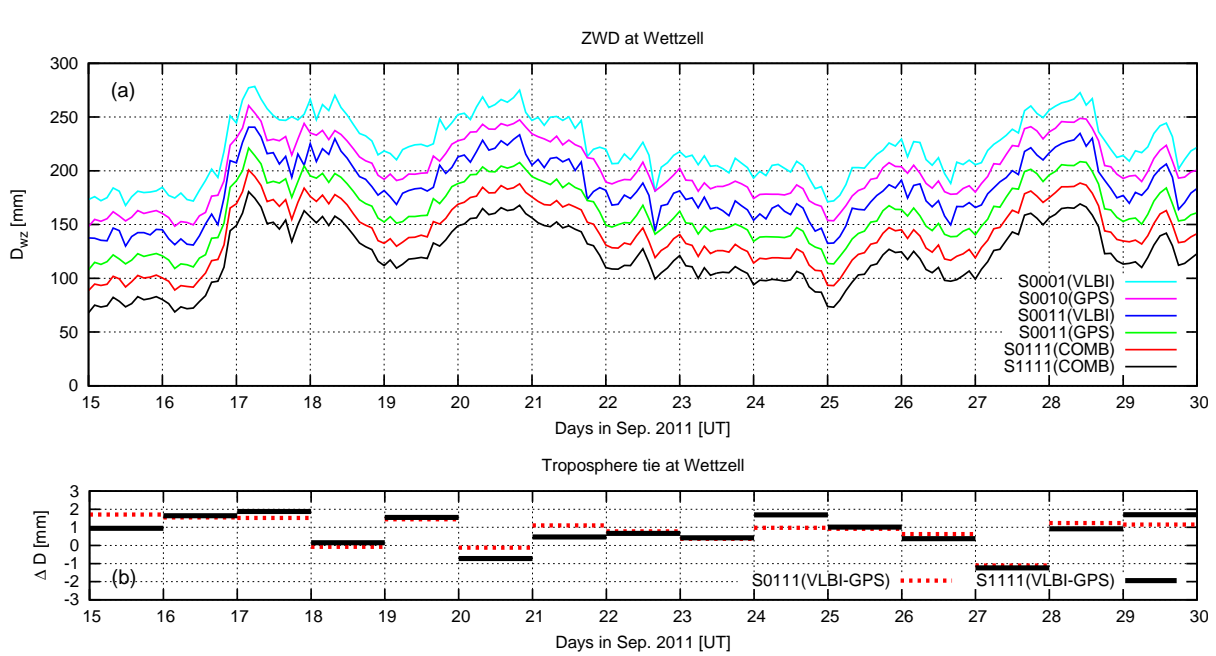

Fig. 4 (a) Estimated zenith wet delays at Wettzell, Germany are plotted over the 15 days CONT11 period. The different solutions are offset by $20 \mathrm{~mm}$ each for better readability. (b) Daily estimates of the troposphere tie between VLBI and GPS at station Wettzell. 


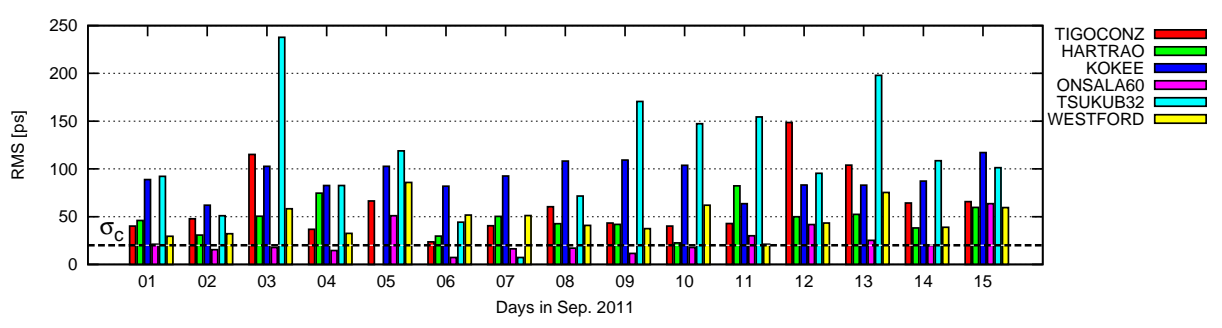

Fig. 5 Daily RMS of detrended three-hourly estimated clock ties. The dotted line (denoted by $\sigma_{c}$ ) shows the average 1-sigma formal error of these ties. Since WTZZ/WETTZELL has been chosen as clock-reference, no clock tie is available for this site. A common clock was not estimated for Station HRAO/HARTRAO on Sep. 5th, 2011 since the GPS receiver clock jumped by one millisecond, whereas the VLBI clock did not show this behavior. 

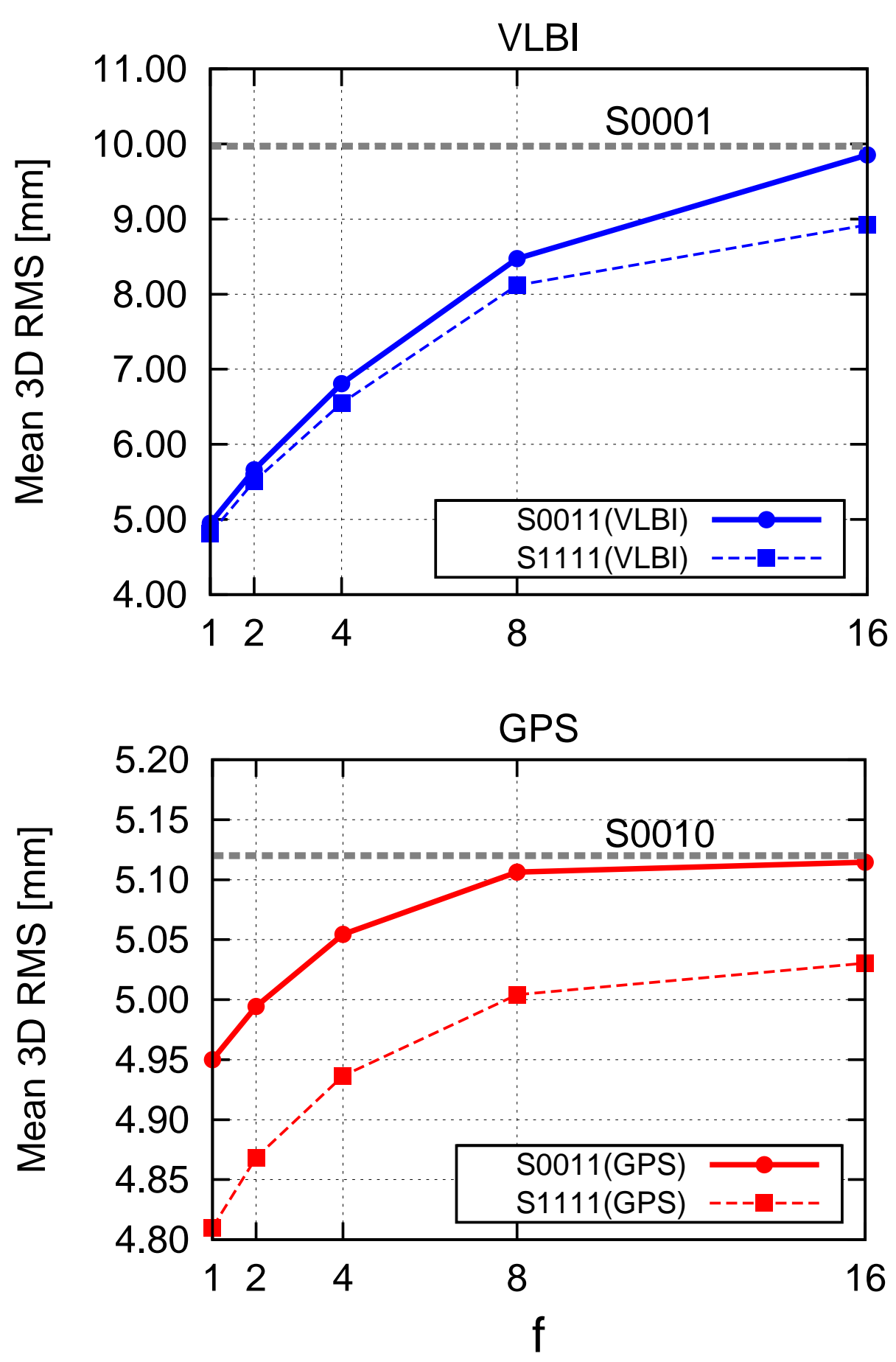

Fig. 6 Mean station position repeatabilities (in $\mathrm{mm}$ ) from solutions S0011 and S1111 which were computed with different multiplicative constants, i.e. $f=1,2,4,8,16$ (cf. Sec. 2.1.1), which scale the formal errors of the local ties. The upper plot shows the mean 3D station position RMS of the VLBI sites together with the corresponding single-technique (solution S0001) performance (dashed line). The lower plot depicts the mean 3D station position RMS of the GPS sites together with the GPS-only solution (S0010) performance (dashed line). 
Table 1 Parameter settings of single- and multi-technique solutions. For solutions S0111 and S1111 a daily troposphere tie was estimated between VLBI and GPS at each site. In solution S1111 the inter-technique clock-offset $\Delta L(t)$ was estimated in the form of a $3 \mathrm{~h}$ piece-wise linear function.

\begin{tabular}{|c|c|c|c|c|c|c|c|c|c|}
\hline \multirow{2}{*}{$\begin{array}{c}\text { solution } \\
\text { name }\end{array}$} & \multirow{2}{*}{$\begin{array}{l}\text { techniques } \\
\text { involved }\end{array}$} & \multirow{2}{*}{$\begin{array}{l}\text { local ties } \\
\text { applied }\end{array}$} & \multicolumn{2}{|c|}{ ZWD } & \multicolumn{2}{|c|}{ gradients } & \multicolumn{2}{|c|}{ clock } & \multirow[t]{2}{*}{ EOPs } \\
\hline & & & GPS & VLBI & GPS & VLBI & GPS & VLBI & \\
\hline S0001 & VLBI only & no & & $2 \mathrm{~h}$ & & $6 \mathrm{~h}$ & & $1 \mathrm{~h}$ & yes \\
\hline S0010 & GPS only & no & $2 \mathrm{~h}$ & & $6 \mathrm{~h}$ & & $5 \mathrm{~min}$ & & no \\
\hline S0011 & GPS+VLBI & yes & $2 \mathrm{~h}$ & $2 \mathrm{~h}$ & $6 \mathrm{~h}$ & $6 \mathrm{~h}$ & $5 \min$ & $1 \mathrm{~h}$ & yes \\
\hline S0111 & GPS+VLBI & yes & & & & & $5 \min$ & $1 \mathrm{~h}$ & yes \\
\hline S1111 & GPS+VLBI & yes & & & & & & & yes \\
\hline
\end{tabular}


Table 2 Summary of the geophysical models used in this study.

\begin{tabular}{|c|c|c|}
\hline Model & VLBI & GPS \\
\hline Solid earth tides & \multicolumn{2}{|c|}{ IERS 2010 conventions (Petit and Luzum, 2010) } \\
\hline Ocean loading & \multicolumn{2}{|c|}{ Multi-mission altimetry model EOT11a (ftp://ftp.dgfi.badw.de/pub/EOT11a) } \\
\hline Atmospheric pressure loading & \multicolumn{2}{|l|}{$\mathrm{S}_{1}-\mathrm{S}_{2}$ corrections (Ray and Ponte, 2003) } \\
\hline EOPs & \multicolumn{2}{|c|}{ A-priori UT1 and polar motion from IERS C04 series } \\
\hline A-priori troposphere delays & \multicolumn{2}{|c|}{ Zenith hydrostatics delays from GPT2 mapped with GMF2 (Lagler et al. 2013) } \\
\hline Wet troposphere delays & \multicolumn{2}{|c|}{ Estimated with wet GMF2 (Lagler et al. 2013) mapping function } \\
\hline Gradients & \multicolumn{2}{|c|}{ Estimated with Chen and Herring (1997) mapping function } \\
\hline Ionosphere & First-order dual-frequency correction & $\begin{array}{l}\text { First order dual-frequency correction and higher order } \\
\text { corrections according to IERS } 2010 \text { conventions }\end{array}$ \\
\hline Antenna phase center & & IGS ANTEX information (igs08_1785.atx) \\
\hline
\end{tabular}


Table 3 Comparison between GPS-VLBI station vector determined from single-technique analysis (i.e. solutions S0001 and S0010) and the terrestrial local ties provided from the IERS.

\begin{tabular}{|c|c|c|c|c|}
\hline \multicolumn{2}{|c|}{ station } & $\Delta x$ & $\Delta y$ & $\Delta z$ \\
\hline \multirow{3}{*}{ CONZ } & $\mathrm{c} 5++$ & $-46.6605 \pm 0.0054$ & $50.2659 \pm 0.0106$ & $-98.5984 \pm 0.0111$ \\
\hline & local tie & $-46.6560 \pm 0.0009$ & $50.2726 \pm 0.0008$ & $-98.6173 \pm 0.0011$ \\
\hline & diff. & $0.0045 \pm 0.0055$ & $0.0067 \pm 0.0106$ & $-0.0189 \pm 0.0112$ \\
\hline \multirow{3}{*}{ HRAO } & $\mathrm{c} 5++$ & $-90.3018 \pm 0.0087$ & $132.1958 \pm 0.0072$ & $-34.6547 \pm 0.0049$ \\
\hline & local tie & $-90.3001 \pm 0.0020$ & $132.1879 \pm 0.0017$ & $-34.6539 \pm 0.0021$ \\
\hline & diff. & $0.0017 \pm 0.0089$ & $-0.0079 \pm 0.0074$ & $0.0008 \pm 0.0053$ \\
\hline \multirow{3}{*}{ KOKB } & $\mathrm{c} 5++$ & $-0.4872 \pm 0.0083$ & $-19.3977 \pm 0.0048$ & $-42.2420 \pm 0.0056$ \\
\hline & local tie & $-0.5037 \pm 0.0023$ & $-19.4023 \pm 0.0021$ & $-42.2335 \pm 0.0023$ \\
\hline & diff. & $-0.0165 \pm 0.0086$ & $-0.0046 \pm 0.0052$ & $0.0085 \pm 0.0061$ \\
\hline \multirow{3}{*}{ ONSA } & $\mathrm{c} 5++$ & $52.6136 \pm 0.0044$ & $-40.4706 \pm 0.0031$ & $-43.8953 \pm 0.0084$ \\
\hline & local tie & $52.6233 \pm 0.0016$ & $-40.4595 \pm 0.0016$ & $-43.8731 \pm 0.0017$ \\
\hline & diff. & $0.0097 \pm 0.0047$ & $0.0111 \pm 0.0035$ & $0.0222 \pm 0.0086$ \\
\hline \multirow{3}{*}{ TSKB } & $\mathrm{c} 5++$ & $209.5582 \pm 0.0078$ & $-29.7301 \pm 0.0065$ & $216.8749 \pm 0.0073$ \\
\hline & local tie & $209.5487 \pm 0.0009$ & $-29.7242 \pm 0.0009$ & $216.8833 \pm 0.0011$ \\
\hline & diff. & $-0.0095 \pm 0.0079$ & $0.0059 \pm 0.0066$ & $0.0084 \pm 0.0074$ \\
\hline \multirow{3}{*}{ WES2 } & $\mathrm{c} 5++$ & $26.7849 \pm 0.0037$ & $41.0336 \pm 0.0056$ & $30.4688 \pm 0.0052$ \\
\hline & local tie & $26.7960 \pm 0.0051$ & $41.0220 \pm 0.0051$ & $30.4760 \pm 0.0051$ \\
\hline & diff. & $0.0111 \pm 0.0063$ & $-0.0116 \pm 0.0076$ & $0.0072 \pm 0.0073$ \\
\hline \multirow{3}{*}{ WTZZ } & $\mathrm{c} 5++$ & $39.6690 \pm 0.0051$ & $117.7088 \pm 0.0030$ & $-60.4137 \pm 0.0061$ \\
\hline & local tie & $39.6737 \pm 0.0001$ & $117.7098 \pm 0.0001$ & $-60.4151 \pm 0.0001$ \\
\hline & diff. & $0.0047 \pm 0.0051$ & $0.0010 \pm 0.0030$ & $-0.0014 \pm 0.0061$ \\
\hline
\end{tabular}


Table 4 Mean station position repeatabilities (in $\mathrm{mm}$ ) from the five different solutions.

\begin{tabular}{c|cccc} 
sol. & $\mathrm{N} / \mathrm{S}$ & $\mathrm{E} / \mathrm{W}$ & $\mathrm{U} / \mathrm{D}$ & $3 \mathrm{D}$ \\
\hline $\mathrm{S} 0001$ & 2.74 & 4.65 & 8.11 & 9.97 \\
$\mathrm{~S} 0010$ & 1.46 & 2.35 & 4.12 & 5.12 \\
$\mathrm{~S} 0011$ & 1.40 & 2.24 & 4.00 & 4.95 \\
$\mathrm{~S} 0111$ & 1.33 & 2.13 & 3.93 & 4.82 \\
$\mathrm{~S} 1111$ & 1.28 & 2.07 & 3.97 & 4.81
\end{tabular}


Table 5 Mean troposphere ties (and their formal errors) between VLBI and GPS. The right column lists the corresponding empirical values from Teke et al. (2011).

\begin{tabular}{c|ccr} 
IGS & S0111 & S1111 & emp. \\
name & {$[\mathrm{mm}]$} & {$[\mathrm{mm}]$} & {$[\mathrm{mm}]$} \\
\hline CONZ & $5.0 \pm 1.9$ & $4.9 \pm 1.8$ & 3.1 \\
HRAO & $-1.1 \pm 2.0$ & $-0.7 \pm 1.7$ & -0.5 \\
KOKB & $4.0 \pm 2.3$ & $4.0 \pm 2.0$ & -2.7 \\
ONSA & $-4.1 \pm 1.6$ & $-4.0 \pm 1.6$ & -4.2 \\
TSKB & $-8.2 \pm 4.5$ & $-8.5 \pm 2.9$ & -6.1 \\
WES2 & $-4.0 \pm 2.0$ & $-3.9 \pm 1.9$ & -0.6 \\
WTZZ & $0.8 \pm 0.7$ & $0.8 \pm 0.9$ & -0.9
\end{tabular}

